\title{
A Swollen Nose - Nasal Septal Hematoma
}

\author{
Ashwin Chandran ${ }^{1} \cdot$ Pirabu Sakthivel $^{1}$ (D) $\cdot$ Chirom Amit Singh ${ }^{1}$
}

Received: 16 July 2019 / Accepted: 31 July 2019 / Published online: 23 August 2019

(C) Dr. K C Chaudhuri Foundation 2019

A 6-y-old boy presented to Otorhinolaryngology emergency clinic with a 1-day history of bilateral nasal obstruction, mouth breathing and pain on the dorsum of the nose. The symptoms occurred after a blunt trauma to the nose due to an accidental fall from bed. Examination revealed a bilaterally symmetrical, bluish, fluctuant swelling in the anterior part of the nasal septum, completely obstructing both the nasal cavities (Fig. 1), along with nasal tip tenderness. The findings were classical of a nasal septal hematoma. Rest of the systemic examination was unremarkable. The child underwent incision and drainage under general anesthesia and merocele packs were inserted for $3 \mathrm{~d}$. Antibiotic cover against Staphylococcus aureus was given for $5 \mathrm{~d}$. The child remained well at 4mo follow-up visit.

A nasal septal hematoma occurs when blood collects in the space between the septal cartilage and the overlying mucoperichondrium $[1,2]$. It is classically bilateral and follows nasal trauma, although bleeding disorders, violent sneezing and anticoagulant drugs like aspirin and warfarin are also attributed to its development. The buckling force strips the perichondrium off the cartilage, tearing the submucosal blood vessels, resulting in a hematoma $[1,2]$. If not drained immediately, the cartilage may undergo necrosis as it is deprived of its nutrition, leading to saddle nose deformity, supra tip collapse and columellar retraction. Undue delay in diagnosis and treatment can also lead to septal abscess, septal perforation and direct spread of infection to cavernous sinus via emissary veins [3]. Hence, all patients with nasal trauma should be evaluated for septal hematoma, and immediate surgical drainage along with nasal packing for 2-3 d (to prevent recurrence) is warranted to avoid complications.

Pirabu Sakthivel

pirabusakthivel@gmail.com

1 Department of Otorhinolaryngology \& Head and Neck Surgery, All India Institute of Medical Sciences, New Delhi 110029, India

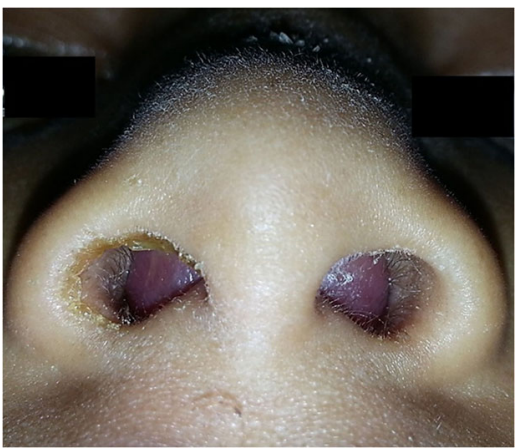

Fig. 1 Clinical image depicting the classical bilateral symmetrical bluish swelling involving the septum

Authors' Contribution AC, PS and CAS were involved in the clinical care of the patient, literature review, and manuscript preparation. All authors read and approved the final manuscript. CAS is the guarantor for this article.

\section{Compliance with Ethical Standards}

Statement of Human and Animal Rights This case image complies with the ethical standards outlined in the journal. It involved the medical management of a child as per society guidelines and was in accordance with the ethical standards of the institution. This case was not formal research involving Human participants and/or Animals.

Informed Consent Informed written consent was obtained from the child father for publication of the clinical image.

Conflict of Interest None.

\section{References}

1. Dispenza C, Saraniti C, Dispenza F, Caramanna C, Salzano FA. Management of nasal septal abscess in childhood: our experience. Int J Pediatr Otorhinolaryngol. 2004;68:1417-21.

2. Alshaikh N, Lo S. Nasal septal abscess in children: from diagnosis to management and prevention. Int J Pediatr Otorhinolaryngol. 2011;75:737-44.

3. Alvarez H, Osorio J, De Diego JI, Prim MP, De La Torre C, Gavilan J. Sequelae after nasal septum injuries in children. Auris Nasus Larynx. 2000;27:339-42.

Publisher's Note Springer Nature remains neutral with regard to jurisdictional claims in published maps and institutional affiliations. 